Draft Version May 29, 2018

Preprint typeset using $\mathrm{LAT}_{\mathrm{E}} \mathrm{X}$ style emulateapj v. 12/16/11

\title{
THE EFFECT OF AGN ON THE GLOBAL H I CONTENT OF ISOLATED LOW-MASS GALAXIES
}

\author{
Jeremy D. Bradford ${ }^{1,4}$, Marla C. Geha ${ }^{1}$, Jenny E. Greene ${ }^{2}$, Amy E. Reines ${ }^{3}$, Claire M. Dickey ${ }^{1}$ \\ 1 Astronomy Department, Yale University, New Haven, CT 06520, USA; marla.geha@yale.edu \\ 2 Department of Astrophysical Sciences, Princeton University, Princeton, NJ 08544, USA and \\ 3 Department of Physics, Montana State University, Bozeman, MT 59717, USA \\ Draft version May 29, 2018
}

\begin{abstract}
We investigate the global neutral hydrogen (H I) content of isolated galaxies selected from the SDSS spectroscopic survey with optical evidence of Active Galactic Nuclei (AGN). Our sample includes galaxies with unresolved H observations from the ALFALFA $70 \%$ data release combined with deeper $\mathrm{H}$ I observations of low-mass galaxies with $7.0<\log \mathrm{M}_{*} / \mathrm{M}_{\odot}<9.5$. We examine the $\mathrm{H}$ I masses of this sample using the distance from the star-forming sequence on the $[\mathrm{O}$ III $] / \mathrm{H} \beta$ and $[\mathrm{N}$ II] $/ \mathrm{H} \alpha$ Baldwin Phillips Terlevich (BPT) diagram as a measurement of AGN activity. In agreement with previous studies, we find that, for galaxies with $\log \mathrm{M}_{*} / \mathrm{M}_{\odot}>9.5$, AGN activity does not correlate with the global H I content. However, for galaxies with $9.2<\log \mathrm{M}_{*} / \mathrm{M}_{\odot}<9.5$, we identify a set of objects at large distances from the BPT star-forming sequence and lower than expected H I masses. This gas-depleted sample is red in both g-r and NUV-r colors and compact without distinguishable signs of star formation. This is surprising because the vast majority of isolated galaxies in this stellar mass regime are both star-forming and gas-rich. These galaxies are greater than $1.5 \mathrm{Mpc}$ from any massive galaxy, ruling out environmental processes as a source of the gas-depletion. We suggest that either black hole feedback or shocks from extremely bursty star formation cause the emission lines and have destroyed or otherwise consumed the cold gas.

Subject headings: galaxies: active galaxies: evolution galaxies: nuclei radio lines: galaxies -galaxies: dwarf
\end{abstract}

\section{INTRODUCTION}

Feedback from active galactic nuclei (AGN) is often invoked as a mechanism to regulate galaxy evolution (e.g., Silk \& Rees 1998; Fabian 1999, Granato et al. 2001, 2004, Di Matteo et al. 2005 Dashyan et al. 2018). AGN feedback, either alone or together with feedback from star formation, can have profound effects on galaxy evolution by heating, ionizing and expelling gas from the interstellar medium (ISM). However, important aspects of AGN feedback physics are poorly constrained and simulations are often tuned to reproduce observations and galaxy scaling relations (e.g., Bower et al. 2006, Somerville et al. 2008, Gabor et al. 2011; DeBuhr et al. 2011; Schaye et al. 2014; Genel et al. 2014).

Studies of the H I content of active galaxies consistently produce results that AGN activity is not directly correlated with the global H I gas mass of galaxies (e.g., Ho et al. 2008; Fabello et al. 2011, Zhu \& Wu 2015). For example, Geréb et al. (2015) find no relation between AGN [O III] luminosity and the global H I content of massive galaxies, even when the AGN are split by red sequence and green valley. However, Lemonias et al. (2014) find that high-mass galaxies with the largest gas fractions and the lowest H I surface densities (and therefore the lowest star formation rate surface densities) are also likely to have signatures of AGN emission and large bulges. While AGN may affect cold gas locally, the above studies support the conclusion that AGN activity is not entirely responsible for the global $\mathrm{HI}$ gas regulation in massive galaxies.

Recent studies have uncovered a large sample of low-

${ }^{4}$ NSF Graduate Research Fellow mass galaxies with $\log \mathrm{M}_{*} / \mathrm{M}_{\odot}<9.5$ showing signatures of accreting black holes (e.g., Reines et al. 2013; Sartori et al. 2015, Reines \& Comastri 2016). Low-mass galaxies offer an opportunity to examine AGN feedback processes in galaxies with shallower gravitational potential wells, where feedback processes may have a more profound effect on the global ISM than in high-mass galaxies. However, low-mass galaxies are strongly affected by environmental processes (e.g., Geha et al. 2012). To clearly examine the properties of galaxies with evidence of AGN, we study isolated galaxies where the effects of environment are minimized.

Bradford et al. (2015, Paper I) showed that isolated galaxies with $\log \mathrm{M}_{*} / \mathrm{M}_{\odot}<9.5$ cannot completely destroy their own cold gas reservoirs. Galaxies with $9.0<$ $\log \mathrm{M}_{*} / \mathrm{M}_{\odot}<9.5$ have gas fractions greater than $20 \%$ and galaxies with $\log \mathrm{M}_{*} / \mathrm{M}_{\odot}<9.0$ have gas fractions greater than $30 \%$. However, flux-limited surveys of $\mathrm{HI}$ emission are, by definition, biased towards gas-rich galaxies and may not contain low-mass galaxies that have been significantly depleted of their H I gas due to feedback processes (Koribalski et al. 2004, Giovanelli et al. 2005).

In this paper, we ask whether the presence of an AGN significantly affects the global H I content of low mass galaxies $\left(\log \mathrm{M}_{*} / \mathrm{M}_{\odot}<9.5\right)$. We compliment the $\mathrm{H}$ I observations from Paper I and data from the ALFALFA H I survey with new, deeper H I observations of low-mass galaxies with evidence of AGN activity. In $\S 2$, we present the galaxy catalog, emission line diagnostic data, H I observations, and catalog statistics. We measure a new $M_{\mathrm{H} \text { I versus }} \mathrm{M}_{*}$ relation for all isolated ALFALFA galaxies and explore emission line diagnostics by measuring the perpendicular distance from the star forming region 
in $[\mathrm{O} \mathrm{III}] / \mathrm{H} \beta$ and $[\mathrm{N} \mathrm{II}] / \mathrm{H} \alpha$ space. In $\S 3$, we examine in detail the $\mathrm{H}$ I gas masses of isolated, low-mass galaxies with evidence of gas-depletion due to AGN activity and compare these galaxies to a gas-normal sample with similar positions on the $[\mathrm{O}$ III $] / \mathrm{H} \beta$ and $[\mathrm{N}$ II $] / \mathrm{H} \alpha$ BPT diagram. We examine these populations and compare them to the rest of the isolated sample. In $\S 4$, we discuss our results and posit what may cause the emission line ratios and gas-depletion in this gas-depleted sample. In this work, we adopt the following cosmological parameters: $\Omega_{0}=0.3, \Omega_{\Lambda}=0.7, H_{0}=70 \mathrm{~km} \mathrm{~s}^{-1}$ (i.e., $\mathrm{h}=$ $0.7)$.

\section{DATA AND METHODS}

\subsection{Galaxy Catalog and Environment Definitions}

We select galaxies from version 0.1.2 of the NASA Sloan Atlas (NSA) catalog ${ }^{2}$ (Blanton et al. 2011). The NSA catalog is based on the Sloan Digital Sky Survey DR8 (SDSS) (Aihara et al. 2011). Stellar masses are generated from the Blanton \& Roweis (2007) kcorrect software and are calculated using a Chabrier (2003) IMF. We assume a 0.2 dex uncertainty in $\mathrm{M}_{*}$. We restrict our galaxy sample to the NSA redshift between 0.002 and 0.055 and we apply several quality cuts as detailed in Paper I. These cuts ensure accurate photometry, spectroscopy and heliocentric distance measurements.

Isolated galaxies have various definitions (Haynes \& Giovanelli 1984, Karachentsev et al. 2011; Brough et al. 2013 Hirschmann et al. 2013). Geha et al. (2012) find that low-mass galaxies with $\log \mathrm{M}_{*} / \mathrm{M}_{\odot}<9.0$ located at least $1.5 \mathrm{Mpc}$ from a massive galaxy are always star-forming and isolated quenched galaxies with $9.0<\log \mathrm{M}_{*} / \mathrm{M}_{\odot}<9.5$ are extremely rare. We adopt this empirically motivated isolation criterion for lowmass galaxies. Isolated low-mass galaxies are therefore defined as $\log \mathrm{M}_{*} / \mathrm{M}_{\odot}<9.5$ with projected distances to a massive host $\left(\log M_{*} / M_{\odot}>10\right)$ greater than $1.5 \mathrm{Mpc}$. Isolated high-mass galaxies are defined as in Paper I with $\log \mathrm{M}_{*} / \mathrm{M}_{\odot}>9.5$, a projected distance larger than $1.5 \mathrm{Mpc}$ to the nearest galaxy more massive than 0.5 dex, and a fifth nearest neighbor surface density $\Sigma_{N}<1 \mathrm{Mpc}^{-2}$. After restricting the galaxies from the NSA catalog to isolated galaxies only, we obtain 36708 isolated galaxies, of which 28621 are high-mass and 8074 are low-mass.

\subsection{H I Observations}

\subsubsection{Our HI Observations}

We have obtained new observations of 30 galaxies using the Arecibo Observatory between March of 2015 and January of 2017. The 21-cm observations used the same configuration detailed in Paper I: L-band Wide receiver using 1024 resolution channels with a bandwidth of 12.5 $\mathrm{MHz}$ and a velocity resolution of $2.6 \mathrm{~km} \mathrm{~s}^{-1}$. Position switched $\mathrm{ON} / \mathrm{OFF}$ observing and ON/OFF noise-diode calibration was used. Our deeper integration times run from 5 to 60 minutes on-source, with an average integration time of 16 minutes. We integrate until a detection has been made or we reach an on-source exposure time of 30 minutes (although in a few cases the exposure time is less due to observing constraints).

\footnotetext{
2 http://www.nsatlas.org
}

For this work, we re-reduce all H I data from Paper I and our new data in order to co-add all spectra from the same receiver configuration and to maximize the number of usable observations. Most of the observations from Paper I focus on isolated galaxies with $\log \mathrm{M}_{*} / \mathrm{M}_{\odot}<9$ and a Sersic axis ratio less than 0.65 in order to obtain accurate kinematics from $\mathrm{H}$ I line-widths. Our new observations focus mostly on the Reines et al. (2013) "dwarf" galaxy sample with signs of AGN activity. We have updated the Paper I H I measurement algorithm to salvage more noise-only spectra and spectra with unstable baselines outside of the spectral window of interest. The most significant update to this algorithm is an adaptive Hanning smoothing length (between 2.5 and $10 \mathrm{~km} / \mathrm{s}$ ) to optimize the measured emission line parameters. Uncertainties in the properties of the $\mathrm{H}$ I emission line are calculated through the same bootstrap process as described in Paper I.

We calculate upper limits for 65 non-detections in this H I sample. Non-detections are identified using a smoothed $\mathrm{S} / \mathrm{N}$ cutoff below 6 and verified by eye. For each non-detection, we estimate the the H I $20 \%$ linewidth using the stellar Tully-Fisher relation from Bradford et al. (2016) and de-project this velocity based on the optical inclination. We then calculate the expected $\mathrm{M}_{\mathrm{H} \text { I }}$ using the stellar mass-to-gas mass relation in $\mathrm{Pa}-$ per I and convert this gas mass to $\mathrm{H}$ I flux. The specific relationship between $\mathrm{M}_{*}$ and $\mathrm{M}_{\mathrm{H} \text { I }}$ we use does not affect our results, as the upper limits are always significantly less than the predicted $\mathrm{M}_{\mathrm{HI}}$ from this relation. We create a synthetic Gaussian emission line with this H I line width and integrated flux. We measure the rms of the observation using the un-smoothed spectrum. We then iterate over this fiducial signal, add in a random noise realization, and Hanning smooth this synthetic spectrum to a resolution of $10 \mathrm{~km} \mathrm{~s}^{-1}$. For each noise realization, we decrease the integrated flux of the synthetic spectrum until we reach the smoothed $\mathrm{S} / \mathrm{N}$ cutoff of 6 . When the $\mathrm{S} / \mathrm{N}$ drops below 6 , we perform 1000 additional noise realizations using this final synthetic emission line. The median integrated flux of these random realizations where the $\mathrm{S} / \mathrm{N}$ is less than 6 is the final upper limit.

We detail our new $\mathrm{HI}$ observations, detections and non-detections, in Table 1. All of these galaxies have emission line measurements from Reines \& Volonteri (2015), which are described in $\S 2.4$. Note that some of these galaxies are isolated and some are not. For the duration of this work we will focus on isolated galaxies only, but we list these non-isolated galaxies for clarity.

\subsubsection{ALFALFA H I Data}

We compliment the $\mathrm{H}$ I data described above with the publicly available ALFALFA 70\% catalog, a blind, driftscan survey (Haynes et al. 2011). We match sources in the ALFALFA catalog using their optical centers with "detcodes" of 1 and 2 to NSA catalog sources within 6 " and where the difference between systemic velocities is less than $75 \mathrm{~km} \mathrm{~s}^{-1}$. We discard any matches to the ALFALFA detcode 2 sources where the $\mathrm{S} / \mathrm{N}$ is less than 6 . For overlapping observations between our data and the ALFALFA catalog, we adopt the error-weighted average of the H I measurements.

For galaxies without a detection that fall within the ALFALFA footprint, we calculate conservative upper 


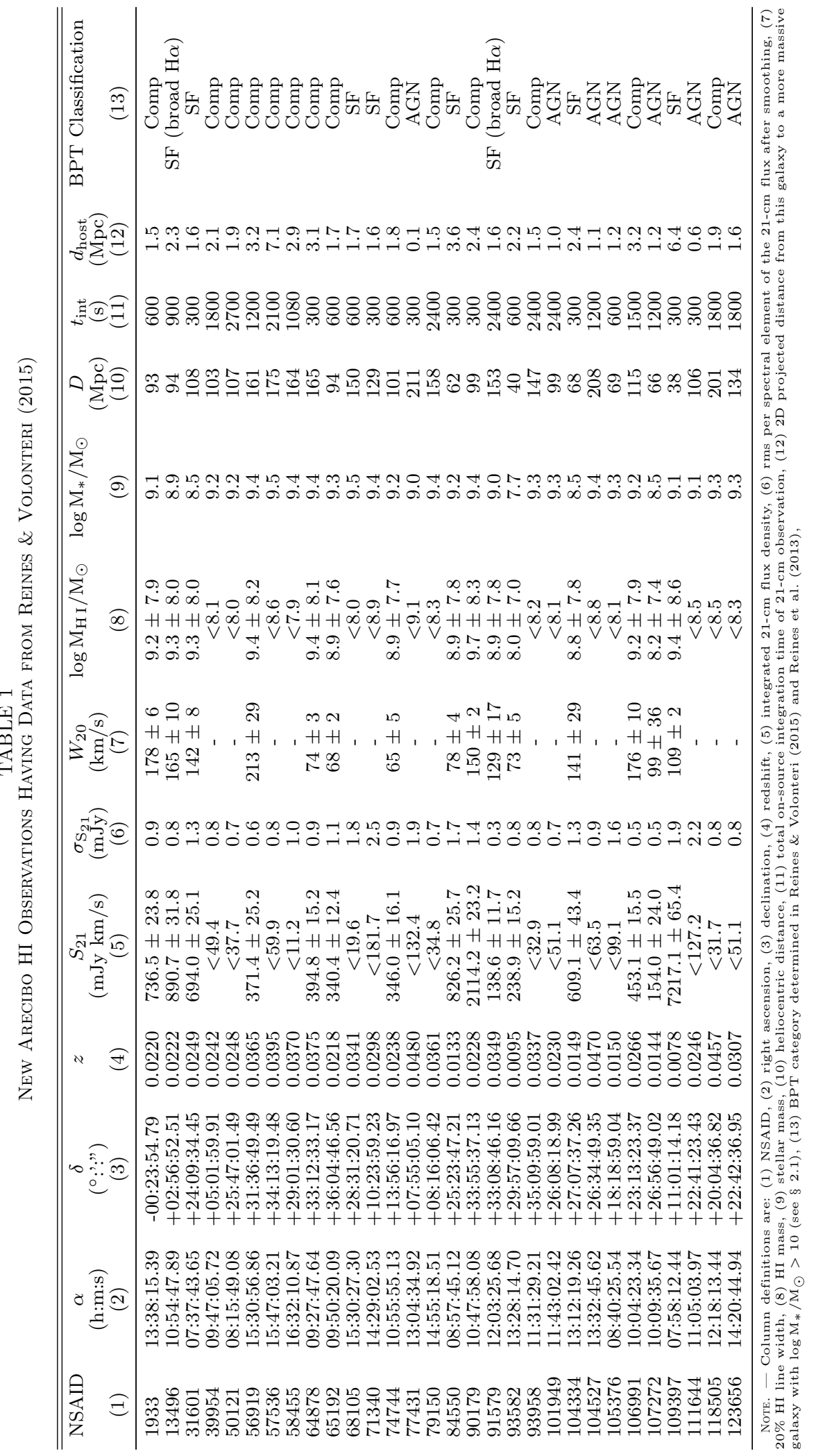




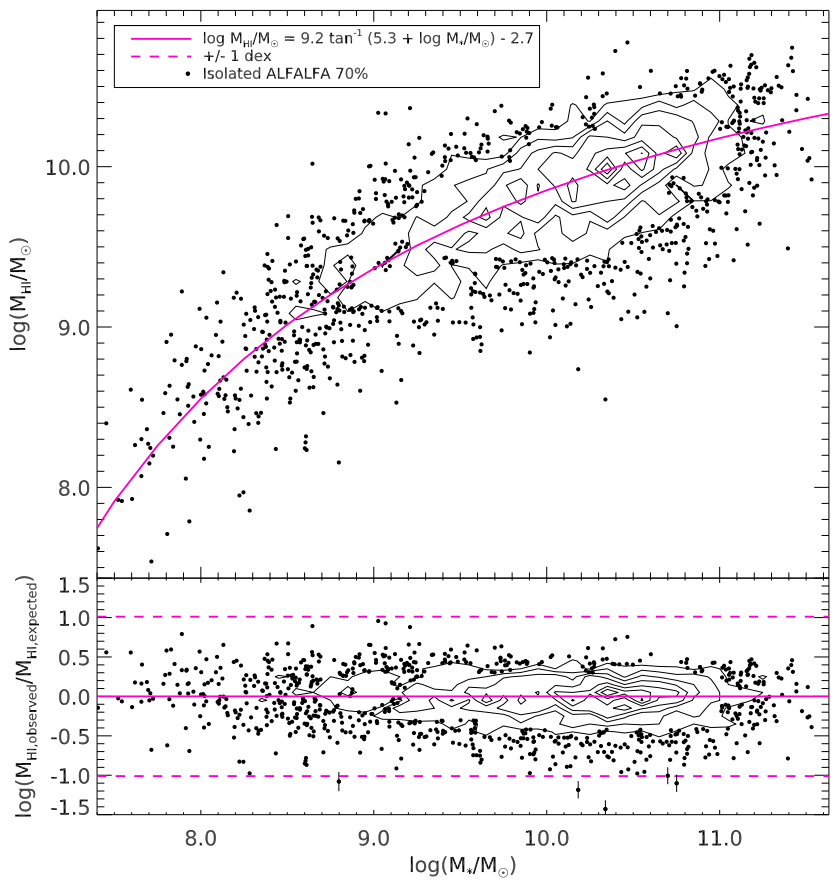

FIG. 1. - Top: The isolated $\mathrm{M}_{\mathrm{H} \text { I }}$ to $\mathrm{M}_{*}$ relation of gas-rich galaxies. Only isolated galaxies with detections from the 70\% ALFALFA survey are plotted here as black contours and dots. The fit from equation 2 is plotted as the solid curve and noted in the legend. Bottom: H I mass residual $\left(\Delta \mathrm{M}_{\mathrm{H} \mathrm{I}}<-1 \mathrm{dex}\right)$ as a function of stellar mass with the same symbols at the top panel. The $\pm 1 \operatorname{dex}(3 \sigma)$ residuals are shown as dashed lines.

limits by using the $\mathrm{S} / \mathrm{N}$ calculation from Haynes et al. (2011) to back out the predicted flux for a S/N of 6 :

$$
S_{21}=\frac{6 \times W_{50} \times \sigma_{\mathrm{rms}}}{w_{\mathrm{smo}}^{1 / 2}},
$$

with $S_{21}$ the HI integrated flux density, $W_{50}$ the $50 \%$ H I line-width, $w_{\text {smo }}=W_{50} / \Delta V_{\text {smooth }}[\mathrm{km} / \mathrm{s}], V_{\text {smooth }}$ is the resolution of the smoothed spectrum and $\sigma_{\text {rms }}$ the measured rms of the Hi spectrum. For this case, $\sigma_{\mathrm{rms}}=2.3 \mathrm{mJy}$, which is the mean rms of the ALFALFA survey. We then calculate the $\mathrm{HI}$ line widths using the stellar Tully-Fisher relation from Bradford et al. (2016) and upper limits as described in $\$ 2.2 .1$.

While we are not guaranteed that this approach accurately represents the upper limits of these galaxies, we find that these upper limits match the ALFALFA Spaenhauer diagram ( $\mathrm{M}_{\mathrm{H}} \mathrm{v}$ versus heliocentric distance) and the H I profile width versus integrated flux density relations from ALFALFA (Figures 3 and 12 in Haynes et al. (2011). These results have motivated us to proceed with these upper limits serving purely as a reference for our primary analysis.

\subsection{The Isolated $\mathrm{M}_{\mathrm{H} \text { I }}$ to $\mathrm{M}_{*}$ Relation}

The observed cold gas content of galaxies is closely related to their stellar mass, galaxy color, and stellar surface density (e.g., Catinella et al. 2010, Huang et al.|2012, Paper I). The baryon content of isolated low-mass galaxies is often dominated by cold gas, likely because galaxy formation is inefficient at low masses (Roychowdhury et al. 2014). A break in the relation between $\mathrm{M}_{\mathrm{H} \text { I }}$ and $M_{*}$ occurs near $\log M_{*}=9.0$, where the gas fraction increases to more than $90 \%$ at the lowest stellar masses. In Paper I, we fit a broken power law to the $M_{\text {gas }}=1.4 M_{\mathrm{H} \mathrm{I}}$ to $\mathrm{M}_{*}$ relation with a break at $\log \mathrm{M}_{*}=8.6$. In this work however, we find that an arctangent function provides a better fit simultaneously to the high and low-mass ends of the $\mathrm{M}_{\mathrm{H} \text { I }}$ to $\mathrm{M}_{*}$ relation while maintaining the break between high and low $M_{*}$ regimes. This function is not physically motivated but offers a better fit to the locus of the distribution in $\mathrm{M}_{\mathrm{H} \text { I }}$ at fixed $\mathrm{M}_{*}$ than a simple broken power-law.

We fit the relation only to the isolated galaxies selected from the NSA catalog that have ALFALFA H I data. The relation is measured with ALFALFA data only because of homogeneity and the survey's well-studied completeness and detection limits (Haynes et al. 2011, § 6). We use only isolated galaxies to minimize the effects of environmental gas-depletion processes on this relationship.

To perform the fit, we bin the data in stellar mass and then fit to the weighted medians of the distribution of $\mathrm{M}_{\mathrm{H} \mathrm{I}}$ in each stellar mass bin. Because the distribution of $\mathrm{M}_{\mathrm{H} \text { I }}$ as a function of stellar mass is log-normal with the tail of the distribution trending to smaller $\mathrm{M}_{\mathrm{H}}$, this fitting method removes any spurious trends in the residuals in this relation, which is critical for our analysis below. We measure the following relation:

$$
\log \mathrm{M}_{\mathrm{HI}} / \mathrm{M}_{\odot}=9.2 \tan ^{-1}\left(5.3+\log \mathrm{M}_{*} / \mathrm{M}_{\odot}\right)-2.7 .
$$

We calculate the residuals from the $\mathrm{M}_{\mathrm{H} \text { I }}$ to $\mathrm{M}_{*}$ relation for both the ALFLFA sample and our H I data as,

$$
\Delta \mathrm{M}_{\mathrm{HI}}=\log \mathrm{M}_{\mathrm{HI}, \mathrm{observed}} / \mathrm{M}_{\mathrm{HI} \text {,expected }} .
$$

We add 0.1 dex in quadrature to all $\mathrm{HI}$ residual uncertainties and upper limits due to the uncertainty in the fit.

We present the relation between $\mathrm{M}_{\mathrm{HI}}$ and $\mathrm{M}_{*}$ in the top panel of Figure 1 for isolated galaxies with ALFALFA data and the residuals $\left(\Delta \mathrm{M}_{\mathrm{HI}}\right)$ between the observed $\mathrm{M}_{\mathrm{H} \text { I }}$ and the predicted $\mathrm{M}_{\mathrm{H} \text { I }}$ in the bottom panel. Equation 2 is plotted as the solid curve. The dashed lines in the bottom panel are \pm 1 dex from the relation, which roughly corresponds to the $3 \sigma$ scatter. We use the -1 dex residual as a reference point throughout the paper as an arbitrary line of demarcation.

\subsection{Emission Line Measurements}

Optical emission line flux ratio diagnostics are often used to differentiate between various physical phenomena that ionize the interstellar medium of galaxies (Baldwin et al. 1981; Veilleux \& Osterbrock 1987). Differentiating between excitation sources (actively accreting black holes, star formation, shocks from AGB stars, etc.) depends on the ionization mechanism strength. Reines \& Volonteri (2015) measure emission line strengths of $\tilde{6} 7,000$ galaxies selected from the NSA catalog. The authors cull the spectral catalog with a S/N cutoff of 3 and an equivalent width cutoff of 1 for the $\mathrm{H} \alpha$, [N II], [O III] emission lines and a $\mathrm{S} / \mathrm{N}$ cutoff of 2 for the $\mathrm{H} \beta$ line. They iteratively subtract continuum and absorption lines using the models of Tremonti et al. (2004) from the observed spectrum, carefully masking out the emission 


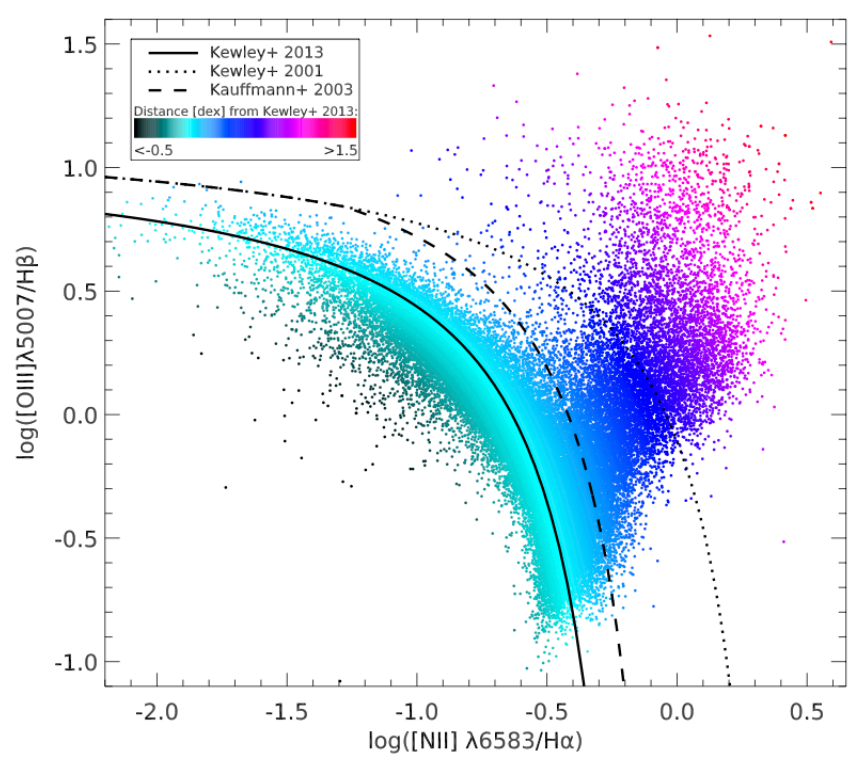

FIG. 2.- $[\mathrm{O} \mathrm{III}] / \mathrm{H} \beta$ versus $[\mathrm{N} \mathrm{II}] / \mathrm{H} \alpha$ emission line diagnostics BPT diagram with data from Reines \& Volonteri (2015), regardless of whether the galaxy has HI data or not. The dotted line is the Kewley et al. (2001) demarcation line, the dashed line is the Kauffmann et al. (2003) demarcation line and the solid line marks the Kewley et al. (2013) star-forming main sequence. Galaxies are color-coded by the perpendicular distance from the star-forming region as shown in the color bar.

lines. Next, a one- or two-component Gaussian is fit to the [S II] doublet and used as a template for the remaining emission lines of interest, excluding [O III]. We use the dataset from this study without any modifications.

\subsection{BPT emission line Diagnostics}

We examine the emission line diagnostics of all galaxies from Reines \& Volonteri (2015), regardless of environment or $\mathrm{HI}$ data using the $[\mathrm{O} \mathrm{III}] / \mathrm{H} \beta$ and $[\mathrm{N} \mathrm{II}] / \mathrm{H} \alpha$ narrow-line BPT diagram (Baldwin et al. 1981, Veilleux \& Osterbrock 1987; Kewley et al. 2006). We adopt the division into the star-forming, composite, and AGN categorization that has been performed by Reines \& Volonteri (2015) using the Kewley et al. (2001) and Kauffmann et al. (2003) categorization. The Kewley et al. (2001) demarcation line represents the division between a theoretical maximal starburst model and emission that can only be explained by AGN activity. The Kauffmann et al. (2003) demarcation line is an empirical separation between the star forming sequence and the AGN sequence. Galaxies below the Kauffmann et al. (2003) line are considered to be purely star forming and galaxies above the Kewley et al. (2001) line are considered Seyferts and LINERs. The region between these two demarcation lines are defined as composite spectra with both star formation and AGN emission.

Galaxies are commonly categorized using these divisions in BPT space. This classification scheme has been calibrated with galaxies more massive than the Reines et al. (2013) sample. For low mass galaxies where AGN emission is weaker and may be more difficult to disentangle from star formation emission, it is unclear where the standard definitions are effective. Lower mass galaxies are also, on average, more metal-poor which will affect the measured line ratios. To further compound this problem, the ratio of emission from star formation to AGN activity is a strong function of the spatial resolution of the observations - where single fiber observations can create aperture bias (e.g., Sharp \& Bland-Hawthorn 2010 Pracy et al. 2014, Gomes et al. 2016). The mean fraction of the surface area covered by the SDSS fiber compared to the $50 \%$ Petrosian radii is $22 \%$ and $32 \%$ for isolated high and low-mass galaxies, respectively. We expect smaller, less massive galaxies with AGN emission to shift towards the composite or possibly the star-forming region of the BPT diagram. Thus emission-line categories may significantly limit our analysis.

It is therefore enlightening to examine galaxy properties relative to position on a BPT diagram as opposed to categorizations (e.g., Kewley et al. 2001; Kauffmann et al. 2003; Kewley et al. 2013 Zhu \& Wu 2015). For example, Kauffmann et al. (2003) examine the $[\mathrm{O}$ III] $/ \mathrm{H} \beta$ and $[\mathrm{N}$ II $] / \mathrm{H} \alpha$ narrow-line $\mathrm{BPT}$ space by defining a distance and an angle from a point where the star-forming sequence meets the plume of galaxies with AGN emission. They find that [O III] luminosity, a measure of AGN strength, does not increase with distance from this point, while [O III] luminosity decreases sharply at a characteristic angle.

We measure the perpendicular distance from the starforming sequence of the $[\mathrm{O} \mathrm{III}] / \mathrm{H} \beta$ and $[\mathrm{N} \mathrm{II}] / \mathrm{H} \alpha \mathrm{BPT}$ diagram as defined by Kewley et al. (2013) for each galaxy. The position of the Kewley et al. (2013) fit is determined by local SDSS star-forming galaxies while the shape of the curve is based on data from their photoionization models (see their Figure 1),

$$
\log \left(\frac{[\mathrm{OIII}]}{\mathrm{H} \beta}\right)=\frac{0.61}{\log ([\mathrm{NII}] / \mathrm{H} \alpha)+0.08}+1.1
$$

For each galaxy, we define $d_{\mathrm{BPT}}$ as the minimum perpendicular distance of each galaxy to equation 4. In Figure2 we present the $[\mathrm{O}$ III $]] / \mathrm{H} \beta$ versus $[\mathrm{N} \mathrm{II}] / \mathrm{H} \alpha$ emission line diagnostic BPT diagram. The data are color-coded by their distance from the star-forming sequence as defined above in equation 4. This figure includes every galaxy with BPT data, regardless of whether or not there exists H I data.

\subsection{Final Sample Description}

For the remainder of this work, we focus on a galaxy sample defined by the following criteria:

1. Galaxies selected from the NSA catalog passing the quality cuts from Paper I. The NSA includes galaxies in the redshift range $0.002<z<0.055$.

2. Galaxies are isolated given the criteria for low-mass and high-mass galaxies given in $\S 2.1$.

3. Galaxies have H I observations from Paper I, our $\mathrm{H}$ I dataset or from the ALFALFA $70 \%$ catalog.

4. Galaxies have well-measured emission line ratios from Reines \& Volonteri (2015).

The sample defined by combining the above criteria is not complete, but does fully sample the BPT diagram. This final data set consists of 2855 isolated galaxies with H I and BPT data, 867 of which we refer to as 'low-mass' $\left(\log \mathrm{M}_{*} / \mathrm{M}_{\odot}<9.5\right)$. 


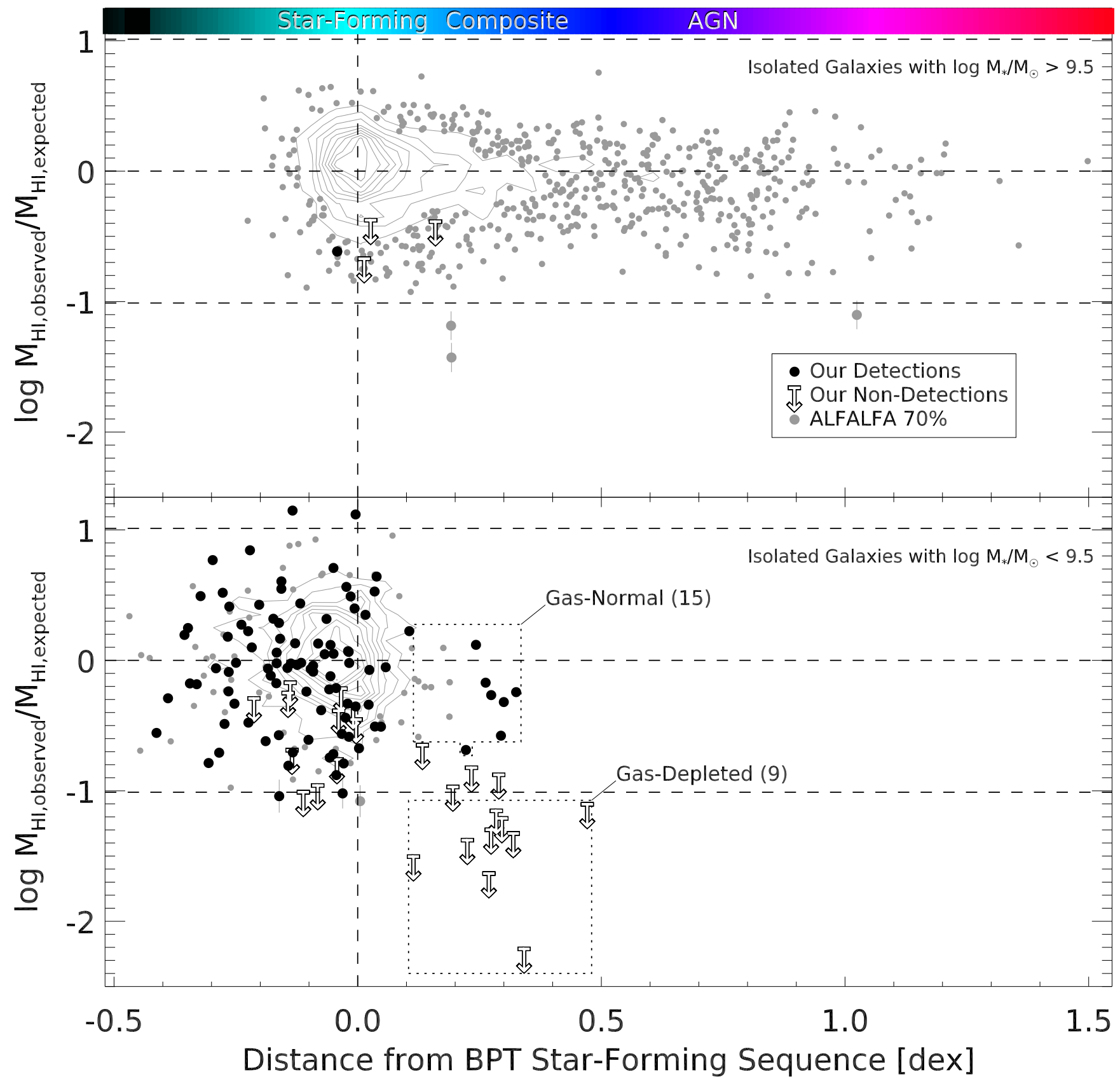

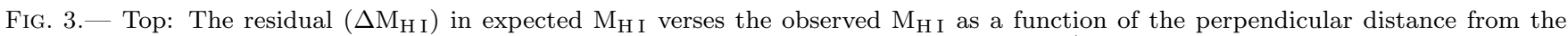
BPT star-forming sequence defined in equation 4 for isolated, high-mass galaxies with $\log \mathrm{M}_{*} / \mathrm{M}_{\odot}>9.5$. Our observations are shown as black dots and our upper limits are shown as downward-pointing arrows. ALFALFA observations are shown as grey dots and contours. The color bar corresponds to the $d_{\mathrm{BPT}}$ as in Figure 2 The star-forming, composite, and AGN labels on the color bar represent the hard cutoff regions in the $[\mathrm{O} \mathrm{III}] / \mathrm{H} \beta$ versus $[\mathrm{N} \mathrm{II}] / \mathrm{H} \alpha$ emission line diagnostics BPT diagram as defined by Kauffmann et al. (2003) and Kewley et al. (2004). Dashed horizontal and vertical lines mark the zeros of both axes. The additional horizontal lines at \pm 1 dex represent the $3 \sigma$ scatter in the fitted relation in $\mathrm{M}_{\mathrm{H}}$ v versus $\mathrm{M}_{*}$ as in the bottom panel of Figure 1 Bottom: The same as the top panel but for isolated low-mass galaxies with $\log \mathrm{M}_{*} / \mathrm{M}_{\odot}<9.5$. In this stellar mass regime, we identify 9 gas-depleted galaxies in the isolated sample with intermediate BPT distances. We also identify a sample of gas-normal galaxies that have been detected in H I at similar $d_{\mathrm{BPT}}$ as the gas-depleted sample. We do not include the non-detections in the intermediate region in our analysis.

\section{RESULTS}

3.1. H I Residuals as a Function of BPT Distance

In Figure 3, we present isolated galaxies with both $\mathrm{BPT}$ and $\mathrm{HI}$ data. We divide this sample at $\log \mathrm{M}_{*} / \mathrm{M}_{\odot}=9.5$, as shown in the top and bottom panels. In this figure, we examine the $\mathrm{M}_{\mathrm{H}}$ residual $\left(\Delta \mathrm{M}_{\mathrm{HI}}\right)$ as a function of distance from the BPT star-forming sequence $\left(d_{\mathrm{BPT}}\right)$. ALFALFA detections are shown as grey points and contours. The HI data from Paper I and 
our deeper observations are shown as black dots for detections and downward pointing arrows for upper limits. The color bar corresponds to $d_{\mathrm{BPT}}$ values as in Figure 2. The star-forming, composite, and AGN regions, as defined by Kewley et al. (2001) and Kauffmann et al. (2003), are labeled in the color bar at the median positions of the $d_{\mathrm{BPT}}$ distributions of the entire sample for each of the three categories. These labels are only meant to act as a guide. The $\pm 3 \sigma$ scatter in the $\mathrm{H}$ I residual, $\Delta \mathrm{M}_{\mathrm{HI}} \pm-1 \mathrm{dex}$, is shown as horizontal dashed lines. Galaxies with $\Delta \mathrm{M}_{\mathrm{H} \text { I }}<-1$ dex are plotted with error bars. Given the conservative ALFALFA upper limit estimates from $\S 2.2 .2$, we would not expect ALFALFA to detect a significant number of galaxies below the $\Delta \mathrm{M}_{\mathrm{HI}}=-1$ dex line.

The high-mass galaxy sample in the top panel of Figure 3 shows no trend in $\mathrm{M}_{\mathrm{H} \text { I }}$ as a function of $d_{\mathrm{BPT}}$. While this is certainly an incomplete $\mathrm{H}$ I sample due to the fluxlimited nature of the ALFALFA survey, we can safely assume the incompleteness is the same for all galaxies across the entire BPT diagram because $d_{\mathrm{BPT}}$ is not dependent on heliocentric distance. This lack of a trend supports previous studies (see $\S 1$ ) suggesting that the global H I content of high-mass galaxies is not significantly affected by AGN activity.

The low-mass galaxy sample is shown in the bottom panel of Figure 3. This sample suffers from the same H I incompleteness as the high-mass sample but, as in the high-mass case, we do not expect this incompleteness to be a function of $d_{\mathrm{BPT}}$. The peak in the distribution of BPT distances is offset by -0.05 dex from the locus of the star-forming sequence. This offset is likely due to the fact that low-mass galaxies are metal-poor compared to massive galaxies (see Kewley et al. (2013) equation 4). Since the star-forming population of low mass galaxies is offset relative to the typical AGN demarcation lines, this further motivates our decision to examine galaxies are a function of BPT distance $\left(d_{\mathrm{BPT}}\right)$.

In the low-mass regime, we have followed-up many galaxies with deeper H I data from Paper I. In the starforming region, we have randomly sampled this population and do not observe any star-forming galaxies with $\Delta \mathrm{M}_{\mathrm{H} \text { I }}<-1$ dex despite these deeper observations. Indeed, given the results from Paper I, we expect cold gas fractions greater than $20 \%$ for all isolated galaxies with $7.0<\log \mathrm{M}_{*} / \mathrm{M}_{\odot}<9.5$. However our deeper $\mathrm{H}$ I observations, do uncover a population of galaxies at intermediate BPT distances where $d_{\mathrm{BPT}}>0.11$ dex and $\mathrm{HI}$ upper limits $\Delta \mathrm{M}_{\mathrm{H} \text { I }}<-1$ dex. These galaxies are "gasdepleted" relative to galaxies in the same stellar mass range by an order of magnitude and they are inconsistent with the $\mathrm{H}$ I distribution of the star-forming population. This gas-depleted galaxy sample with $\Delta \mathrm{M}_{\mathrm{HI}}<-1$ dex may be indicative of an ionizing process other than starformation that is depleting the ISM of $\mathrm{HI}$.

In the same intermediate region of $\mathrm{BPT}$ distances where $d_{\mathrm{BPT}}>0.11 \mathrm{dex}$, we also find low-mass galaxies that are "gas-normal". These gas-normal galaxies have $\mathrm{HI}$ gas masses that are consistent both with the starforming population and with galaxies in the same stellar mass range. This implies that whatever is causing these strong emission line ratios does not always significantly deplete the global H I mass.

\subsection{Isolated Low-Mass Galaxies at Intermediate BPT Distances}

We find a set of gas-depleted, isolated, low-mass $\left(\log \mathrm{M}_{*} / \mathrm{M}_{\odot}=9.5\right)$ galaxies with signs of AGN activity. We next compare the gas-depleted galaxies at large $d_{\mathrm{BPT}}$ to the gas-normal galaxies in the same $d_{\mathrm{BPT}}$ regime in order to explore the nature of the ionizing power source. We also compare the properties of both the gasdepleted sample and the gas-normal sample to a stellarmass matched sample of isolated galaxies.

In other to further understand the source of ionization in our large $d_{\mathrm{BPT}}$ galaxies, we examine additional line diagnostics. Active galaxies are often split into Seyfert and LINERs using the the $[\mathrm{O} \mathrm{III}] / \mathrm{H} \beta$ and $[\mathrm{S} \mathrm{II}] / \mathrm{H} \alpha$ narrowline BPT diagram (Heckman 1980). While Seyfert emission is associated with accretion onto a black hole, LINER emission is more ambiguous, having been detected outside the nucleus of many galaxies. Kewley et al. (2006) identify a separation $[\mathrm{S} \mathrm{II}] / \mathrm{H} \alpha$ vs $[\mathrm{O} \mathrm{III}] / \mathrm{H} \beta$ BPT space between Seyfert and LINER emission.

In Figure 4 , we present the $[\mathrm{O}$ III $] / \mathrm{H} \beta$ and $[\mathrm{S}$ II $] / \mathrm{H} \alpha$ narrow-line diagram in the left panel and revisit the $\left[\begin{array}{ll}\mathrm{O} & \mathrm{III}\end{array}\right] / \mathrm{H} \beta$ and $[\mathrm{N}$ II $] / \mathrm{H} \alpha$ BPT diagram in the right panel. All galaxies with emission line data are plotted as black points and contours. The gas-depleted, isolated, low-mass sample is shown as red, downward-pointing triangles and the gas-normal, isolated, low-mass sample is shown as blue triangles (see $\S 3.1$ ). In the left panel, the Kewley et al. (2006) demarcation curve separates Seyferts and LINERs from the HII region and the straight line splits the active galaxies into Seyferts and LINER branches, as labeled.

In the left panel of Figure 4 the majority of the gasdepleted and the gas-normal samples lie above and along the extreme starburst curve of Kewley et al. (2001, 2006). Both samples are therefore largely inconsistent with the star-forming galaxies. The gas-depleted sample above the curve is split between the Seyfert and LINER regions, while the gas-normal sample is mostly LINER-like. Figure 4 provides further evidence that the emission line measurements of the majority of the gas-depleted sample and a fraction of the galaxies in the gas-normal sample are partially due to ionization processes other than star formation (see further discussion in $\S 4$ ).

In order to further disentangle the source of the emission line ratios and the relationship of these processes to the global H I content of these galaxies, we examine the galaxy properties of the two samples. In Figure 5 we present SDSS images of the gas-depleted sample. The identifications used in each figure are the NSAIDs from version 0.1 .2 of the NSA catalog. The SDSS fiber positions and sizes are plotted as red circles on each galaxy image. The heliocentric distances are noted in each image. All of the gas-depleted galaxies have similar visual properties (red, compact and nucleated) aside from 35979 and 68105 which are red yet more prolate in their respective orientations than the rest of the gas-depleted sample. The SDSS fiber covers a significant fraction of these galaxies' projected areas and these galaxies' spectra may contain a significant amount of contamination from emission outside of the galaxy centers (see $\S 3.1$ ).

In Figure 6, we present images of the gas-normal sample. This sample is diverse in galaxy properties rela- 


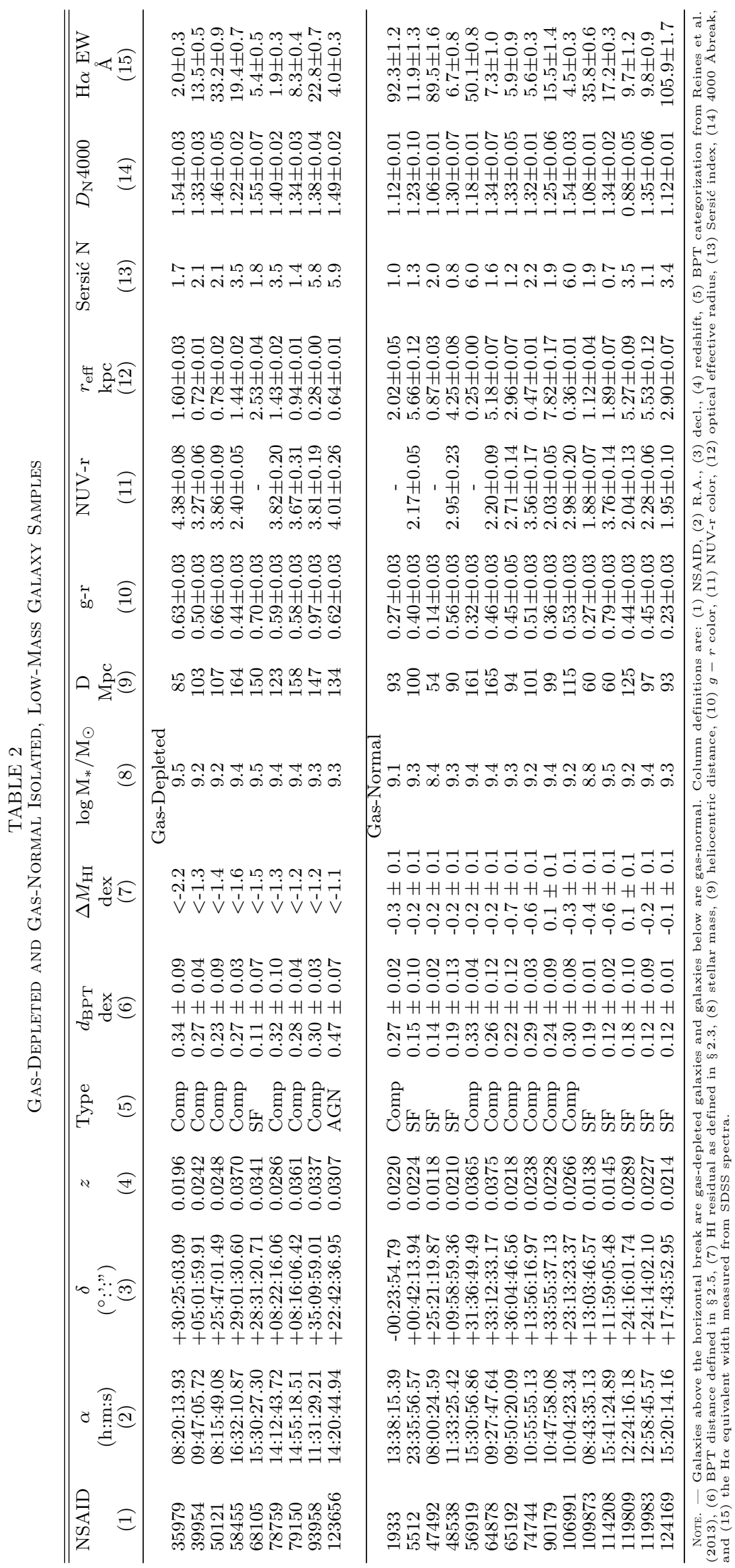




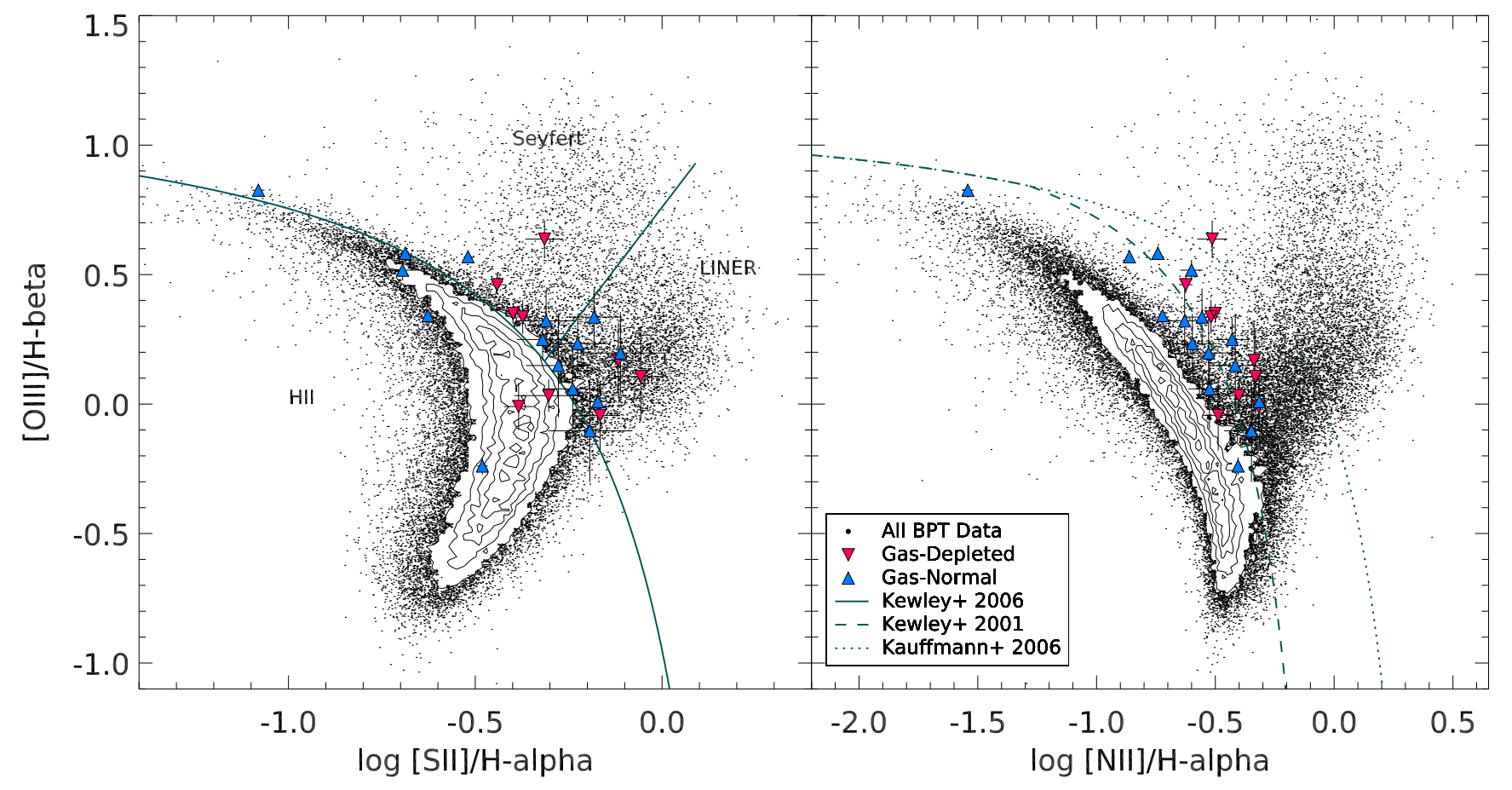

FIG. 4.- Left: The $[\mathrm{O} \mathrm{III}] / \mathrm{H} \beta$ and $[\mathrm{S} \mathrm{II}] / \mathrm{H} \alpha$ narrow-line diagram. All galaxies with BPT data are plotted as black points and contours. The gas-depleted, isolated, low-mass sample is shown as red, downward-pointing triangles and the gas-normal, isolated, low-mass sample is shown as blue triangles (see $\S 3.1$. The Kewley et al. (2006) demarcation between Seyferts and LINERs as well as their separation from the HII region are shown as the green line and curve, respectively. The majority of gas-depleted galaxies are found above the Kewley et al. (2006) demarcation line in both the Seyfert and the LINER regions. The gas-normal sample is similarly distributed, but trends towards the LINER region. The distribution of the two samples support the conclusion that the emission line ratios are due to ionization processes other than star formation. Right: The $[\mathrm{O}$ III] $] / \mathrm{H} \beta \mathrm{vs}[\mathrm{N} \mathrm{II}] / \mathrm{H} \alpha$ BPT diagram. Data and symbols are the same as in the left panel. Curves are those defined as in Figure 2 Note that many of the gas-normal galaxies are located above the locus of the star-forming region but are categorized as "star-forming" using the standard BPT classification scheme. 7 of the 9 gas-depleted galaxies are considered composites, 1 is star-forming and 1 is considered an AGN. 

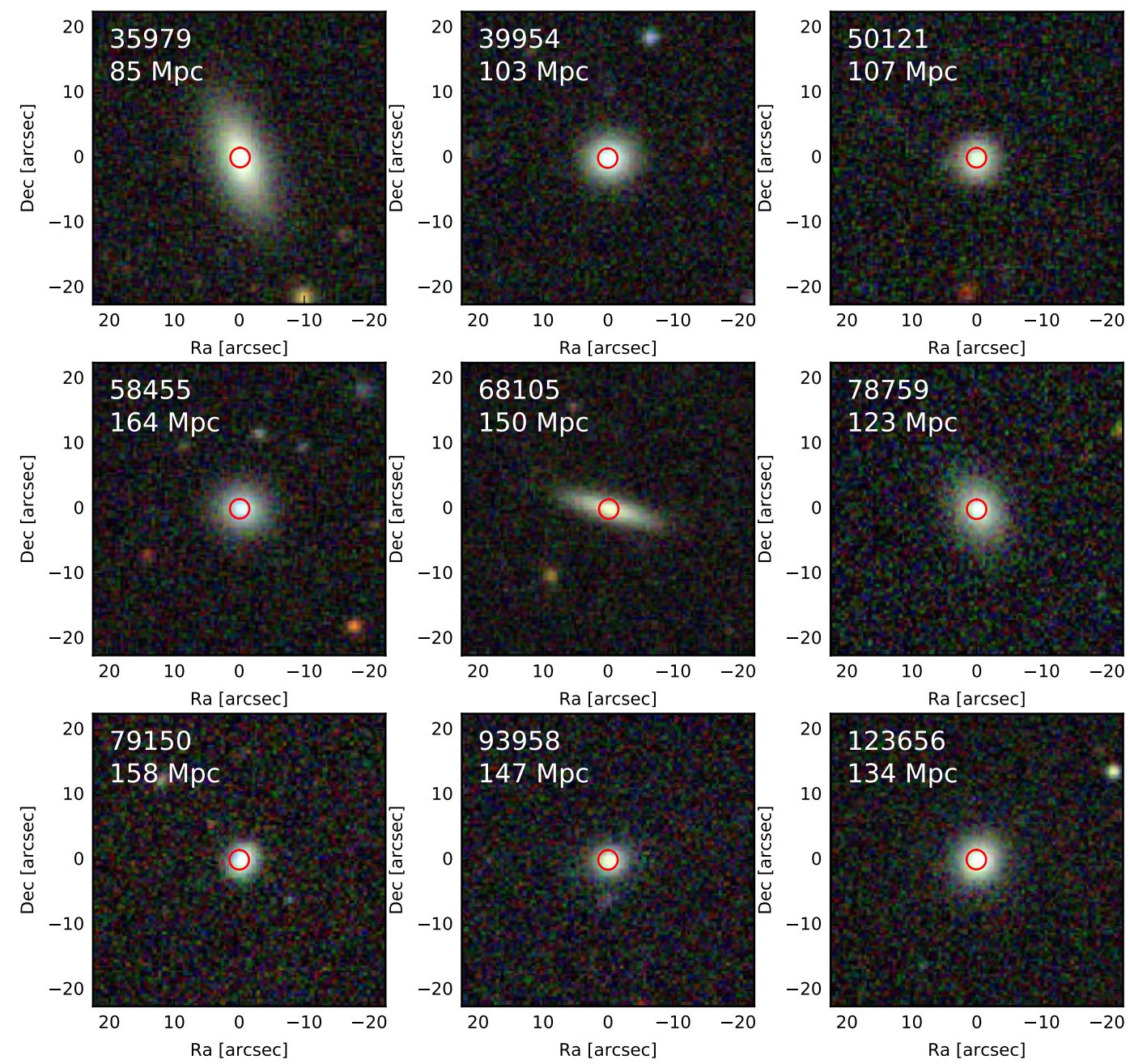

FIG. 5. - SDSS images of gas-depleted, low-mass, isolated galaxies at large BPT distances. The NSAID is noted in the top right of each image. The SDSS fiber position and angular size on the galaxy is shown as a red circle. The heliocentric distance of each galaxy is noted below each NSAID. These gas-depleted galaxies are all red, compact and nucleated. 35979 and 68105 are red, but prolate. These galaxies are visually inconsistent with typical star-forming galaxies.

tive to the gas-depleted sample. Objects 56919, 74744, 106991 and 114208 are similar in appearance to the gasdepleted population and the SDSS fiber again covers a significant fraction of these galaxies. The remaining galaxies tend to be blue and more diffuse with some signs of spiral structure and larger sizes than the SDSS fiber. For many of these galaxies there are visual signs of both diffuse and concentrated star formation regions.

In Figure 7, we examine the distributions of g-r colors, NUV-r colors, effective radius, Sersić index, $\mathrm{D}_{n} 4000$, and $\mathrm{H} \alpha$ equivalent width of both the gas-normal and the gasdepleted low-mass samples relative to all isolated galaxies in the NSA catalog within the same stellar mass range (with $9.2<\log \mathrm{M}_{*} / \mathrm{M}_{\odot}<9.5$ ). The gas-normal sample is shown as upward pointing triangles, color-coded by $\Delta \mathrm{M}_{\mathrm{H} \text { I }}$. The gas-depleted sample is shown as downward pointing triangles, also color-coded by $\Delta \mathrm{M}_{\mathrm{HI}}$. Isolated galaxies from the NSA catalog, excluding the two samples of interest, are shown as grey histograms in each panel regardless of whether BPT or H I data exists. The median of each distribution is shown as a vertical dashed line. We present these and other relevant properties of the gas-depleted and gas-normal galaxy samples in Table 2

Confirming our visual analysis, the distribution of the gas-depleted sample properties is inconsistent with being drawn from the overall star-forming population. For each property shown in Figure 7, we test the null hypothesis that the gas-depleted/gas-normal sample and the full isolated sample of galaxies in the same stellar mass range are drawn from the same underlying population. We use a variation of the Student's t-test without the assumption that the variances of the two populations are equal. The gas-depleted galaxies are not drawn from the same distribution as the overall population with greater $95 \%$ confidence for all 6 properties shown in Figure 7. For the gas-normal population, the distributions are consistent (that is, we cannot reject the null hypothesis that they are drawn from different underlying populations).

We also examine [O III] luminosity in the same fashion as above (not shown). Both the gas-normal and gasdepleted samples are consistent with the general distribution of [O $\mathrm{III}]$ luminosity. However, the general distribution become restricted to galaxies that have emis- 

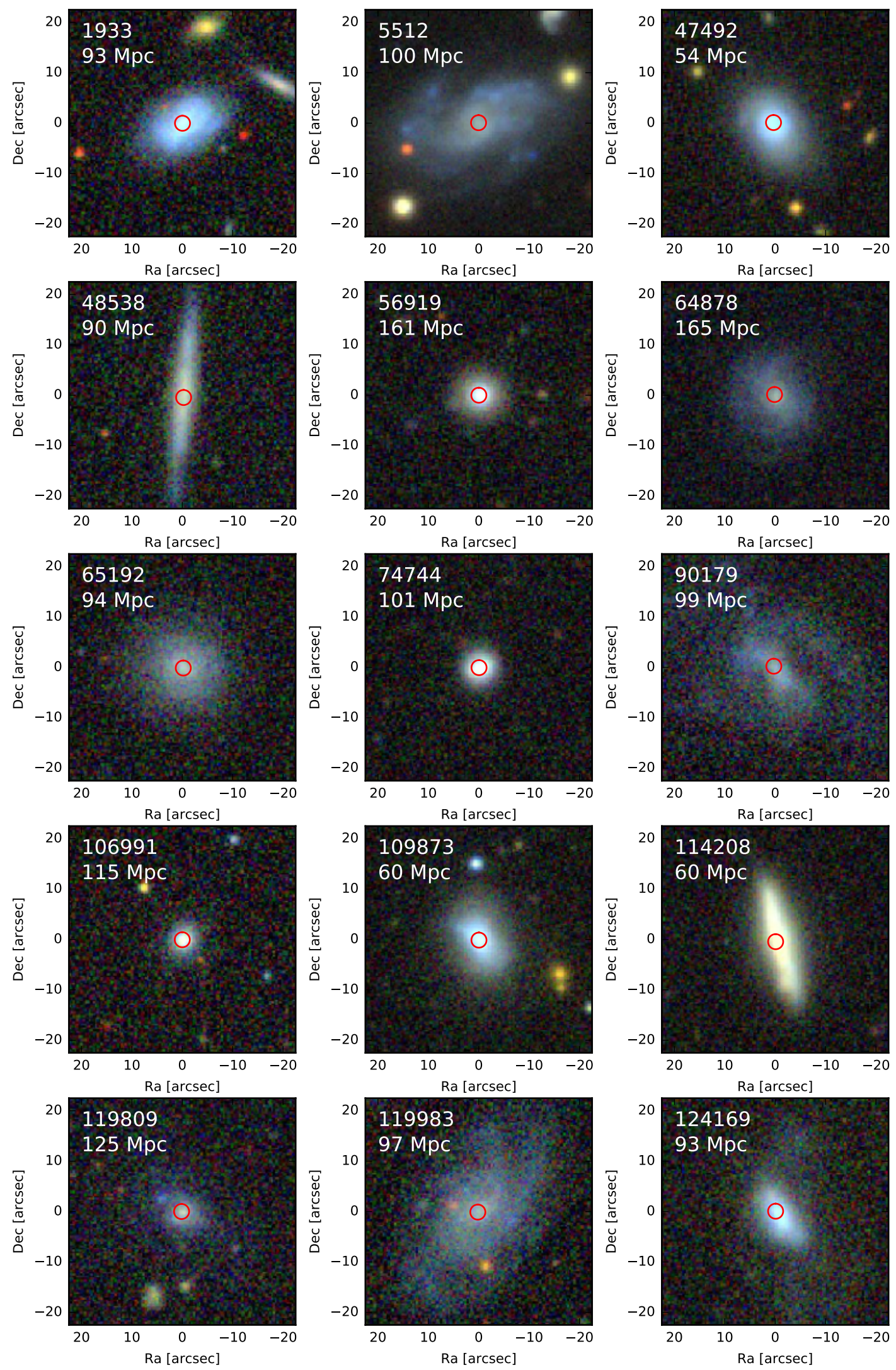

FIG. 6.- Same as in Figure 5 but for the gas-normal low-mass isolated galaxies at large BPT distances. This galaxy population is much more diverse than the gas-depleted sample. Many galaxies are blue and diffuse with some signs of structure and star formation and the SDSS fiber covers a small fraction of these galaxies. 56919, 74744, 106991 and 114208 are all visually similar to the gas-depleted sample. 

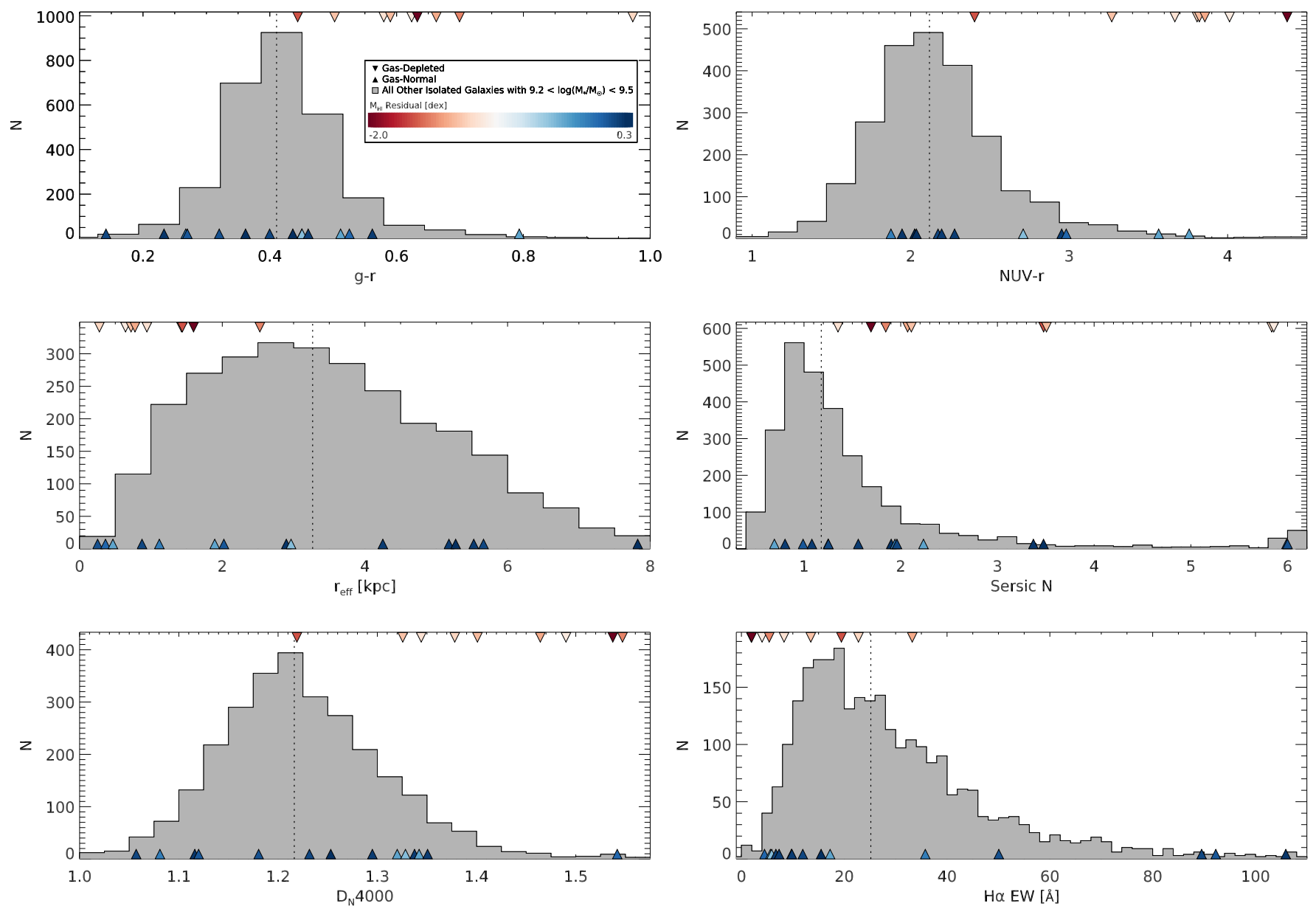

FIG. 7.- Distribution of gas-depleted and gas-normal isolated galaxies relative to the distribution of all isolated galaxies in the cleaned NSA catalog within the stellar mass range $9.2<\log \mathrm{M}_{*} / \mathrm{M}_{\odot}<9.5$. The median of each distribution is shown as a vertical dashed line. Gasdepleted, isolated, low-mass galaxies are identified as downward pointing triangles. Gas-normal, isolated, low-mass galaxies are identified as upward pointing triangles. Both sets of galaxies are color-coded according to their HI mass residual as noted in the color bar. The grey histogram is that of all isolated NSA galaxies within the stellar mass range of the isolated low-mass gas-depleted sample regardless of whether BPT or H I data exist and does not include the gas-depleted or the gas-normal samples. From the top left panel, moving left to right and downward are the g-r colors, NUV-r colors, effective radii, Sersić index, $\mathrm{D}_{n} 4000$ and $\mathrm{H} \alpha$ equivalent widths. Note that we also examine [O III] luminosity (not shown) and both samples are consistent with the general distribution.

sion line measurements from Reines \& Volonteri (2015). Kauffmann et al. (2003) also do not find that [O III] luminosity changes as a function of a galaxy's distance from locus of the star-forming BPT region.

\section{DISCUSSION}

We find a set of galaxies with $9.2<\log \mathrm{M}_{*} / \mathrm{M}_{\odot}<$ 9.5 that are both isolated and gas-poor. These galaxies are located primarily in the composite region of the $[\mathrm{O}$ III $] / \mathrm{H} \beta$ versus $[\mathrm{N}$ II] $/ \mathrm{H} \alpha \mathrm{BPT}$ diagram and the LINER/Seyfert regions of the $[\mathrm{O}$ III $] / \mathrm{H} \beta$ and $[\mathrm{S}$ II $] / \mathrm{H} \alpha$ BPT diagram. These gas-depleted galaxies are also compact and red with older stellar populations and no obvious signs of star formation.

This result is surprising because isolated galaxies in this mass range are generally gas-rich, diffuse, blue and show ongoing star formation. Indeed, Geha et al. (2012) find that less than $2 \%$ of isolated galaxies with $9.2<$ $\log \mathrm{M}_{*} / \mathrm{M}_{\odot}<9.5$ are quiescent. If these galaxies are truly not forming stars, they are extremely rare cases of quenched, low-mass galaxies in isolation. Here we ex- plore the possible physical processes behind the emission line ratios and what may have removed the gas from these galaxies. We note that these galaxies are defined as at least $1.5 \mathrm{Mpc}$ from any massive galaxy, such that the usual environmental processes which dominate in group and cluster environments are unlikely to be active.

A straightforward interpretation is that these galaxies are depleted of H I because of AGN feedback. In this scenario, the line ratios that we observe are excited by accretion onto the central black hole, and energy from accretion has successfully coupled to the gas in the galaxy on large scales and removed and/or destroyed the gas in the galaxy. Accretion energy is observed to drive large scale outflows in molecular (e.g., Alatalo et al. 2011; Veilleux et al. 2013), warm ionized (e.g., Liu et al. 2013; Harrison et al. 2012), and hot (warm absorbers, BALs) gas (e.g., Crenshaw et al. 2003 Reeves et al. 2009). The energy may couple to the gas via radiation pressure on dust (e.g., Thompson et al. 2005), via thermal heating (e.g., Weinberger et al. 2016) or via input of kinetic energy (e.g., Choi et al. 2012). 
Many of the gas-normal and gas-depleted galaxies are classified as LINERs. While LINER emission in massive galaxies has been directly linked with black hole accretion, there are many instances of off-nuclear LINERs (see Ho (2008) and references therein). Even in cases with unambiguous signs of accretion, it is not clear that the AGN provide sufficient photons to photoionize the gas (Eracleous et al. 2010, Yan \& Blanton 2012). A number of photoionization sources may explain the observed line ratios. Shocks and post-AGB (pAGB) stars are possible sources of excitation for the gas. In the case of pAGB stars, we would expect $\mathrm{H} \alpha$ equivalent widths less than $3 \AA$ if pAGB stars are causing a diffuse stellar emission (Binette et al. 1994; Belfiore et al. 2016, 2015). The $\mathrm{H} \alpha$ equivalent width distribution suggests that the majority of the line emission cannot be excited by pAGB stars, leaving shocks or AGN as the most viable options.

If AGN activity is not responsible for heating or removing the cold gas, perhaps the compact structure of the gas-depleted galaxies provides a clue to their nature. Star formation may have been extremely bursty and destroyed or otherwise consumed the cold gas. Compact galaxies can indeed experience extreme outflows caused by compact stellar feedback (Diamond-Stanic et al. 2012). If most of the star-forming cold gas has been removed, we may be seeing the remaining shocked gas from the outflows. This scenario could explain both the gas depletion and the line ratios without invoking an AGN. The three compact, gas-normal galaxies at large BPT distances will be interesting to study for signs of higher star-formation rates or larger outflow velocities than their more diffuse counterparts. Finally, higher spatial resolution imaging and spectroscopy of both the star-forming and gasdepleted compact galaxies would be enlightening, to determine whether they have a diffuse disk component or central point source.

\section{SUMMARY}

In this work, we study the H I masses of galaxies with signs of AGN activity as measured by optical emission lines. We compile a catalog of isolated galaxies with optical emission lines measured from SDSS DR8 spectra and single-dish $\mathrm{H}$ I observations both from the ALFALFA $70 \%$ data release and from Paper I. We obtain new, deeper HI observations with a focus on isolated, low-mass galaxies selected from the Reines et al. (2013) "dwarf" galaxy sample with signs of AGN activity. We measure a new $\mathrm{M}_{*}-\mathrm{M}_{\mathrm{H} \text { I }}$ relation using ALFALFA $70 \%$ data as in Equation 2. We also measure the distance of each galaxy with $[\mathrm{O} \mathrm{III}] / \mathrm{H} \beta$ and $[\mathrm{N} \mathrm{II}] / \mathrm{H} \alpha$ data from the Baldwin Phillips Terlevich (BPT) star-forming sequence $\left(d_{\mathrm{BPT}}\right)$. We use this distance as a measurement of AGN activity strength and compare $\mathrm{M}_{\mathrm{H} \text { I }}$ as a function of $d_{\mathrm{BPT}}$. Our results are summarized as follows:

1. We identify a set of isolated, low-mass galaxies with $\log \mathrm{M}_{*} / \mathrm{M}_{\odot}<9.5$ and $d_{\mathrm{BPT}}>0.11$ dex that is significantly gas-depleted.

2. 7 of the 9 gas-depleted galaxies are located above the $\mathrm{H}$ II demarcation line of Kewley et al. (2006) on the $[\mathrm{O} \mathrm{III}] / \mathrm{H} \beta$ and $[\mathrm{S} \mathrm{II}] / \mathrm{H} \alpha$ diagram, 3 of which are categorized as LINERs and 4 as Seyferts.

3. The gas-depleted, low-mass, isolated galaxies with large $d_{\mathrm{BPT}}$ are red and compact with old stellar populations and no distinguishable signs of star formation. The property distributions of the gasdepleted sample are inconsistent with the general population of isolated galaxies in the same stellar mass range.

4. We compare the gas-depleted sample to a population of 15 gas-normal, low-mass, isolated galaxies with large $d_{\mathrm{BPT}}$. This sample consists primarily of star-forming, late-type galaxies. The majority of these galaxies are consistent with the general population of isolated galaxies in the same stellar mass range. However, we find 3 gas-normal galaxies with similar visual and structural properties as the gas-depleted population. We find a similar fraction of these gas-normal galaxies in the LINER and Seyfert regions on the $[\mathrm{O}$ III $] / \mathrm{H} \beta$ and $[\mathrm{S} \mathrm{II}] / \mathrm{H} \alpha \mathrm{BPT}$ diagram as the gas-depleted sample.

Because these galaxies are very isolated (more distance than $1.5 \mathrm{Mpc}$ from a more massive neighbor), environmental process cannot explain the emission line ratios or H I masses in the gas-depleted sample. We suggest two possibilities below.

In the first case, the energy from black hole accretion successfully couples to the cold gas and causes both the emission lines and the depletion of $\mathrm{H}$ I. The combination of deep radio and X-ray data would be useful to test the nature of these sources. As far as we are aware, no observations in radio continuum or X-ray exist for these galaxies.

In the second case, the compact structural properties of the gas-depleted sample may indicate a much more efficient and energetic star formation history than other galaxies in the same stellar mass regime. It could be that the shocks from this star formation generated the emission lines and removed the cold gas from these galaxies. Deeper imaging and resolved spectroscopy may help examine the nature of these compact galaxies. Star formation histories for both the gas-normal and gas-depleted compact galaxies would also help us determine the nature of these galaxies.

We thank Frank van den Bosch and Pieter van Dokkum for comments on this work. J.D.B. acknowledges support from the Gruber Foundation and the National Science Foundation Graduate Research Fellowship Program under Grant No. DGE-1122492. The Arecibo Observatory is operated by SRI International under a cooperative agreement with the National Science Foundation (AST-1100968), and in alliance with Ana G. MéndezUniversidad Metropolitana, and the Universities Space Research Association. The National Radio Astronomy Observatory is a facility of the National Science Foundation operated under cooperative agreement by Associated Universities, Inc. Funding for SDSS-III has been provided by the Alfred P. Sloan Foundation, the Participating Institutions, the National Science Foundation, and the U.S. Department of Energy Office of Science. The SDSS-III web site is http://www.sdss3.org/. 


\section{REFERENCES}

Aihara, H. et al. 2011, ApJS, 193, 29

Alatalo, K., Blitz, L., Young, L. M., Davis, T. A., Bureau, M. Lopez, L. A., Cappellari, M., Scott, N., Shapiro, K. L., Crocker, A. F., Martín, S., Bois, M., Bournaud, F., Davies, R. L., de Zeeuw, P. T., Duc, P. A., Emsellem, E., Falcón-Barroso, J., Khochfar, S., Krajnovic, D., Kuntschner, H., Lablanche, P. Y., McDermid, R. M., Morganti, R., Naab, T., Oosterloo, T., Sarzi, M., Serra, P., \& Weijmans, A. 2011, ApJ, 735, 88

Baldwin, J. A., Phillips, M. M., \& Terlevich, R. 1981, PASP, 93, 5

Belfiore, F., Maiolino, R., Bundy, K., Thomas, D., Maraston, C.,

Wilkinson, D., Sánchez, S. F., Bershady, M., Blanc, G. A., Bothwell, M., Cales, S. L., Coccato, L., Drory, N., Emsellem, E., Fu, H., Gelfand, J., Law, D., Masters, K., Parejko, J., Tremonti, C., Wake, D., Weijmans, A., Yan, R., Xiao, T., Zhang, K., Zheng, T., Bizyaev, D., Kinemuchi, K., Oravetz, D. \& Simmons, A. 2015, MNRAS, 449, 867

Belfiore, F., Maiolino, R., Maraston, C., Emsellem, E., Bershady, M. A., Masters, K. L., Yan, R., Bizyaev, D., Boquien, M., Brownstein, J. R., Bundy, K., Drory, N., Heckman, T. M., Law, D. R., Roman-Lopes, A., Pan, K., Stanghellini, L., Thomas, D., Weijmans, A.-M., \& Westfall, K. B. 2016, MNRAS, 3111

Binette, L., Magris, C. G., Stasinska, G., \& Bruzual, A. G. 1994, A\&A, 292, 13

Blanton, M. R., Kazin, E., Muna, D., Weaver, B. A., \& Price-Whelan, A. 2011, AJ, 142, 31

Blanton, M. R. \& Roweis, S. 2007, AJ, 133, 734

Bower, R. G., Benson, A. J., Malbon, R., Helly, J. C., Frenk, C. S., Baugh, C. M., Cole, S., \& Lacey, C. G. 2006, MNRAS, 370,645

Bradford, J. D., Geha, M. C., \& Blanton, M. R. 2015, ApJ, 809, 146

Bradford, J. D., Geha, M. C., \& van den Bosch, F. C. 2016, ApJ, 832, 11

Brough, S., Croom, S., Sharp, R., Hopkins, A. M., Taylor, E. N., Baldry, I. K., Gunawardhana, M. L. P., Liske, J., Norberg, P., Robotham, A. S. G., Bauer, A. E., Bland-Hawthorn, J., Colless, M., Foster, C., Kelvin, L. S., Lara-Lopez, M. A. Lopez-Sanchez, A. R., Loveday, J., Owers, M., Pimbblet, K. A., \& Prescott, M. 2013, MNRAS, 435, 2903

Catinella, B., Schiminovich, D., Kauffmann, G., Fabello, S., Wang, J., Hummels, C., Lemonias, J., Moran, S. M., Wu, R. Giovanelli, R., Haynes, M. P., Heckman, T. M., Basu-Zych, A. R., Blanton, M. R., Brinchmann, J., Budavári, T., Gonçalves, T., Johnson, B. D., Kennicutt, R. C., Madore, B. F., Martin, C. D., Rich, M. R., Tacconi, L. J., Thilker, D. A., Wild, V., \& Wyder, T. K. 2010, MNRAS, 403, 683 Chabrier, G. 2003, PASP, 115, 763

Choi, E., Ostriker, J. P., Naab, T., \& Johansson, P. H. 2012, ApJ 754,125

Crenshaw, D. M., Kraemer, S. B., \& George, I. M. 2003, ARA\&A, 41, 117

Dashyan, G., Silk, J., Mamon, G. A., Dubois, Y., \& Hartwig, T 2018, MNRAS, 473, 5698

DeBuhr, J., Quataert, E., \& Ma, C.-P. 2011, MNRAS, 420, 2221

Di Matteo, T., Springel, V., \& Hernquist, L. 2005, Nature, 433, 604

Diamond-Stanic, A. M., Moustakas, J., Tremonti, C. A., Coil, A. L., Hickox, R. C., Robaina, A. R., Rudnick, G. H., \& Sell, P. H. 2012, ApJ, 755, L26

Eracleous, M., Hwang, J. A., \& Flohic, H. M. L. G. 2010, ApJ, 711,796

Fabello, S., Kauffmann, G., Catinella, B., Giovanelli, R., Haynes, M. P., Heckman, T. M., \& Schiminovich, D. 2011, MNRAS, 416,1739

Fabian, A. C. 1999, MNRAS, 308, L39

Gabor, J. M., Davé, R., Oppenheimer, B. D., \& Finlator, K. 2011, MNRAS, 417, 2676

Geha, M., Blanton, M. R., Yan, R., \& Tinker, J. L. 2012, ApJ, 757,85

Genel, S., Vogelsberger, M., Springel, V., Sijacki, D., Nelson, D., Snyder, G., Rodriguez-Gomez, V., Torrey, P., \& Hernquist, L. 2014, eprint arXiv: 1405.3749

Geréb, K., Morganti, R., Oosterloo, T. A., Hoppmann, L., \& Staveley-Smith, L. 2015, A\&A, 580, A43

Giovanelli, R. et al. 2005, AJ, 130, 2598

Gomes, J. M. et al. 2016, A\&A, 585, A92

Granato, G. L., De Zotti, G., Silva, L., Bressan, A., \& Danese, L. 2004, ApJ, 600, 580

Granato, G. L., Silva, L., Monaco, P., Panuzzo, P., Salucci, P., De Zotti, G., \& Danese, L. 2001, MNRAS, 324, 757

Harrison, C. M., Alexander, D. M., Swinbank, A. M., Smail, I., Alaghband-Zadeh, S., Bauer, F. E., Chapman, S. C., Del Moro, A., Hickox, R. C., Ivison, R. J., Menéndez-Delmestre, K., Mullaney, J. R., \& Nesvadba, N. P. H. 2012, MNRAS, 426, 1073

Haynes, M. P. \& Giovanelli, R. 1984, AJ, 89, 758
Haynes, M. P. Giovanelli, R. Martin, A. M., Hess, K. M., Saintonge, A., Adams, E. A. K., Hallenbeck, G., Hoffman, G. L., Huang, S., Kent, B. R., Koopmann, R. A., Papastergis, E., Stierwalt, S., Balonek, T. J., Craig, D. W., Higdon, S. J. U., Kornreich, D. A., Miller, J. R., O'Donoghue, A. A., Olowin, R. P., Rosenberg, J. L., Spekkens, K., Troischt, P., \& Wilcots, E. M. 2011, AJ, 142, 170

Heckman, T. M. 1980, A\&A, 87, 152

Hirschmann, M., De Lucia, G., Iovino, A., \& Cucciati, O. 2013, MNRAS, 433, 1479

Ho, L. C. 2008, ARA\&A, 46, 475

Ho, L. C., Darling, J., \& Greene, J. E. 2008, ApJ, 681, 128

Huang, S., Haynes, M. P., Giovanelli, R., \& Brinchmann, J. 2012, ApJ, 756, 113

Karachentsev, I. D., Makarov, D. I., Karachentseva, V. E., \& Melnyk, O. V. 2011, Astrophys. Bull., 66, 1

Kauffmann, G., Heckman, T. M., Simon White, D. M., Charlot, S., Tremonti, C., Brinchmann, J., Bruzual, G., Peng, E. W., Seibert, M., Bernardi, M., Blanton, M., Brinkmann, J., Castander, F., Csabai, I., Fukugita, M., Ivezić, Ž., Munn, J. A., Nichol, R. C., Padmanabhan, N., Thakar, A. R., Weinberg, D. H., \& York, D. 2003, MNRAS, 341, 33

Kewley, L. J., Dopita, M. A., Leitherer, C., Davé, R., Yuan, T., Allen, M., Groves, B., \& Sutherland, R. 2013, ApJ, 774, 100

Kewley, L. J., Dopita, M. A., Sutherland, R. S., Heisler, C. A., \& Trevena, J.' 2001, ApJ, 556, 121

Kewley, L. J., Geller, M. J., \& Jansen, R. A. 2004, AJ, 127, 2002

Kewley, L. J., Groves, B., Kauffmann, G., \& Heckman, T. 2006 MNRAS, 372, 961

Koribalski, B. S., Staveley-Smith, L., Kilborn, V. A., Ryder, S. D., Kraan-Korteweg, R. C., Ryan-Weber, E. V., Ekers, R. D., Jerjen, H., Henning, P. A., Putman, M. E., Zwaan, M. A., de Blok, W. J. G., Calabretta, M. R., Disney, M. J. Minchin, R. F., Bhathal, R., Boyce, P. J., Drinkwater, M. J., Freeman, K. C., Gibson, B. K., Green, A. J., Haynes, R. F., Juraszek, S., Kesteven, M. J., Knezek, P. M., Mader, S., Marquarding, M., Meyer, M., Mould, J. R., Oosterloo, T., O'Brien, J., Price, R. M., Sadler, E. M., Schröder, A., Stewart, I. M., Stootman, F., Waugh, M., Warren, B. E., Webster, R. L., \& Wright, A. E. 2004, AJ, 128, 16

Lemonias, J. J., Schiminovich, D., Catinella, B., Heckman, T. M., \& Moran, S. M. 2014, ApJ, 790, 27

Liu, G., Zakamska, N. L., Greene, J. E., Nesvadba, N. P. H., \& Liu, X. 2013, MNRAS, 436, 2576

Pracy, M. B., Owers, M. S., Zwaan, M., Couch, W., Kuntschner, H., Croom, S. M., \& Sadler, E. M. 2014, MNRAS, 443, 388

Reeves, J. N., O'Brien, P. T., Braito, V., Behar, E., Miller, L., Turner, T. J., Fabian, A. C., Kaspi, S., Mushotzky, R., \& Ward, M. 2009, ApJ, 701, 493

Reines, A. E. \& Comastri, A. 2016, Publ. Astron. Soc. Aust., 33, 1304

Reines, A. E., Greene, J. E., \& Geha, M. 2013, ApJ, 775, 116

Reines, A. E. \& Volonteri, M. 2015, ApJ, 813, 82

Roychowdhury, S., Chengalur, J. N., Kaisin, S. S., \& Karachentsev, I. D. 2014, MNRAS, 445, 1392

Sartori, L. F., Schawinski, K., Treister, E., Trakhtenbrot, B. Koss, M., Shirazi, M., \& Oh, K. 2015, MNRAS, 454, 3722

Schaye, J., Crain, R. A., Bower, R. G.. Furlong, M., Schaller, M., Theuns, T., Dalla Vecchia, C., Frenk, C. S., McCarthy, I. G., Helly, J. C., Jenkins, A., Rosas-Guevara, Y. M., White, S. D. M., Baes, M., Booth, C. M., Camps, P., Navarro, J. F., Qu, Y., Rahmati, A., Sawala, T., Thomas, P. A., \& Trayford,'J. 2014, MNRAS, 446, 521

Sharp, R. G. \& Bland-Hawthorn, J. 2010, ApJ, 711, 818

Silk, J. \& Rees, M. J. 1998, A\&A, 331, L1

Somerville, R. S., Hopkins, P. F., Cox, T. J., Robertson, B. E., \& Hernquist, L. 2008, MNRAS, 391, 481

Thompson, T. A., Quataert, E., \& Murray, N. 2005, ApJ, 630, 167

Tremonti, C. A., Heckman, T. M., Kauffmann, G., Brinchmann, J., Charlot, S., White, S. D. M., Seibert, M., Peng, E. W., Schlegel, D. J., Uomoto, A., Fukugita, M., \& Brinkmann, J. 2004, ApJ, 613, 898

Veilleux, S., Meléndez, M., Sturm, E., Gracia-Carpio, J., Fischer, J., González-Alfonso, E., Contursi, A., Lutz, D., Poglitsch, A., Davies, R., Genzel, R., Tacconi, L., de Jong, J. A., Sternberg, A., Netzer, H., Hailey-Dunsheath, S., Verma, A., Rupke, D. S. N., Maiolino, R., Teng, S. H., \& Polisensky, E. 2013, ApJ, 776,27

Veilleux, S. \& Osterbrock, D. E. 1987, ApJS, 63, 295

Weinberger, R., Springel, V., Hernquist, L., Pillepich, A. Marinacci, F., Pakmor, R., Nelson, D., Genel, S., Vogelsberger, M., Naiman, J., \& Torrey, P. 2016, MNRAS, 465, 3291

Yan, R. \& Blanton, M. R. 2012, ApJ, 747, 61

Zhu, Y.-N. \& Wu, H. 2015, AJ, 149, 10 